BOBO ZHAO, Ph.D.1

(Corresponding author)

E-mail: bbzhao@bjtu.edu.cn

TAO TANG, Ph.D. ${ }^{2}$

E-mail: ttang@bjtu.edu.cn

${ }^{1}$ National Engineering Research Center of Rail

Transportation Operation and Control System

Beijing Jiaotong University

No. 3 Shangyuancun, Xizhimenwai, Haidian District,

Beijing, 100044, China

${ }^{2}$ State Key Laboratory of Rail Traffic Control and Safety

Beijing Jiaotong University

No. 3 Shangyuancun, Xizhimenwai, Haidian District,

Beijing, 100044, China
Human-Transport Interaction Original Scientific Paper Submitted: 10 July 2019 Accepted: 19 Feb. 2020

\title{
EVALUATING THE LEARNING PERFORMANCE OF EMERGENCY PROCEDURES FOR OPERATORS IN METRO BASED ON A COGNITION MODEL
}

\begin{abstract}
With the rapid development of the automated metro, valid emergency procedures play a significant role for operators in metro emergency response and recovery. Also, the operators have a challenge to learn different emergency procedures under different automation grades of the metro. Hence, the paper aims to evaluate the learning performance of emergency procedures with regard to the operator. Based on the ACT-R cognitive theory, two decision patterns of the operators are proposed to predict the operator's learning process for emergency procedures, and a cognition model including 16 production rules and 32 chunks to realize the perceptual encoding and the corresponding determining parts is built. After that, an experiment is further implemented to validate the model results.
\end{abstract}

\section{KEY WORDS}

cognitive modelling; emergency procedures; human performance modelling; rail transit system; levels of automation; trust in automation;

\section{INTRODUCTION}

An increasing number of metro lines are being built, and the new metro lines are being automated in different cities around the world. The automated metro has several advantages, such as cost-effectiveness, high-traffic frequency and flexibility. Under the automated system, the driver's tasks have been gradually replaced by the complex and automatic functions of a control system. However, the operators are required to recognize emergency situations and handle their abnormal aspects. Automated systems are still man-machine systems, and both technical and human factors are important [1].

The emergencies of a metro system could create extremely serious consequences. For example, the Daegu metro fire accident in Korea in February 2003 caused death of 192 people and 151 people were injured. As a result, considerable efforts have been devoted to improve the safety of the automated metro by metro operators and suppliers. Some projects, such as SECUTR-ED [2], MOD safe and Secure Metro [3], which concern organizational and technological measures, have addressed some types of integrated solutions to increase the effectiveness of the responses to emergencies.

Nevertheless, the existing operational experiences have continuously addressed that human performance is one of the dominant factors affecting the safety of the system process [4-8]. During emergencies, the operators are required to follow highly prescribed emergency procedures that have the specific functions to provide correct and complete instructions in an emergency [7]. The emergency procedures play a further decisive role in the operators' practical processes under challenging environments, such as significant time pressure or uncertain conditions [9]. Therefore, it is essential to successfully provide useful emergency procedures for the operators to accomplish their tasks.

Many qualitative guidelines or checklists have been employed to confirm whether emergency procedures satisfy the necessary requirements, such 
as "emergency procedures should be prepared for operators to easily and clearly understand the operational context" or "emergency procedures should be designed appropriately for operators to accomplish their tasks within an acceptable workload" [10-12]. However, a qualitative method has several drawbacks, such as a subjective way of working or ambiguous requirements that cannot give a quantitative evaluation of the emergency procedures.

For the drawbacks of the qualitative methods, some researchers have devoted to applying mockup test technologies on the evaluation of emergency procedures. For example, the emergency procedures for airport transport were tested by a mock-test method to analyse the relative efficiencies of four specific configurations [13]. Nevertheless, the mock-up test method cannot perform the emergency procedures frequently and thoroughly, and it is always time-consuming.

Furthermore, an extensive effort has been made to quantitatively study how the emergency procedures affect human performance. Park et al. [14] proposed a task complexity measure for the operating procedures of nuclear power plants and further validated the appropriateness of this measure by comparing task complexity scores with two sets of operator response time data. In this method, the abstract hierarchy $(\mathrm{AH})$, which considers the amount of knowledge required to recognize the place of a problem in accomplishing the task, is one of the innovate points of the complexity measure for the task [15].

The emergency procedures are designed as stepby-step instructions that can cover all the emergency situations [16]. As the executor of the emergency procedures, the operators play a significant role in handling the emergency process; thus, they should fully understand the context of the emergency procedures. Although the task complexity measure can provide a quantitative evaluation of emergency procedures, it is insufficient in exploring the intrinsic correlation of the human factors and emergency procedures with the cognitive aspects.

Generally, the cognitive theory is presented to provide a powerful tool for understanding how a human thinks and predicting how a human will behave. For this advantageous feature, many studies have applied the cognitive theory to their specific domains. A rigorous computational model of driver behaviour has been explored in a cognitive architecture to predict and recognize the driver's behaviour and distractions [17]. In the nuclear field, a cognitive model of the operator's high-level activities has been employed to describe a situational assessment and response-planning process with the concept of modelling decision-making in an emergency [18-20], and in the aviation domain, a computational cognitive model of pilots has been used to diagnose possible sources of human performance and error prediction [21]. From these studies, it is demonstrated that the cognition theory is a significant contributor to the research of human performance.

The concept of higher automation makes the metro staff to be in a more alert state for emergencies. In other words, a higher grade of metro system automation has more requirements from the operators to emergency procedures. For this reason, the objective of this paper is to discover how the variances of automation grades affect the operators to learn different emergency procedures and how the operators store and cognize the changes with the evolution of the metro system. The remaining part of this paper is organized as follows: Section 2 and Section 3 briefly introduce the cognition theory adopted in this study and the background about the study. After that, a cognitive model based on ACT-R theory, simulating operator knowledge and how operators think about emergency procedures, is proposed in Section 4. Additionally, a GUI object is created to provide the interface of the external world with the cognitive model in Section 5, and in Section 6, a corresponding experiment is designed to validate the result of the cognitive model. The discussions about the comparison between the simulation results and the experimental data are analysed in Section 7. Finally, some conclusions are drawn in Section 8.

\section{BRIEF INTRODUCTION OF ACT-R}

The ACT-R theory was presented by Anderson [22] to model and understand the human higher level of cognition. This theory has been applied to a broad domain such as mathematical problem solving in the classroom, driver behaviour prediction in car driving, and human memory and other tasks. ACT-R is such a comprehensive theory that can model human thinking, receiving and processing of information, as well as the learning and forgetting processes.

There exist two assumptions representing human knowledge in the ACT-R theory: declarative knowledge and procedural knowledge. The former is used 


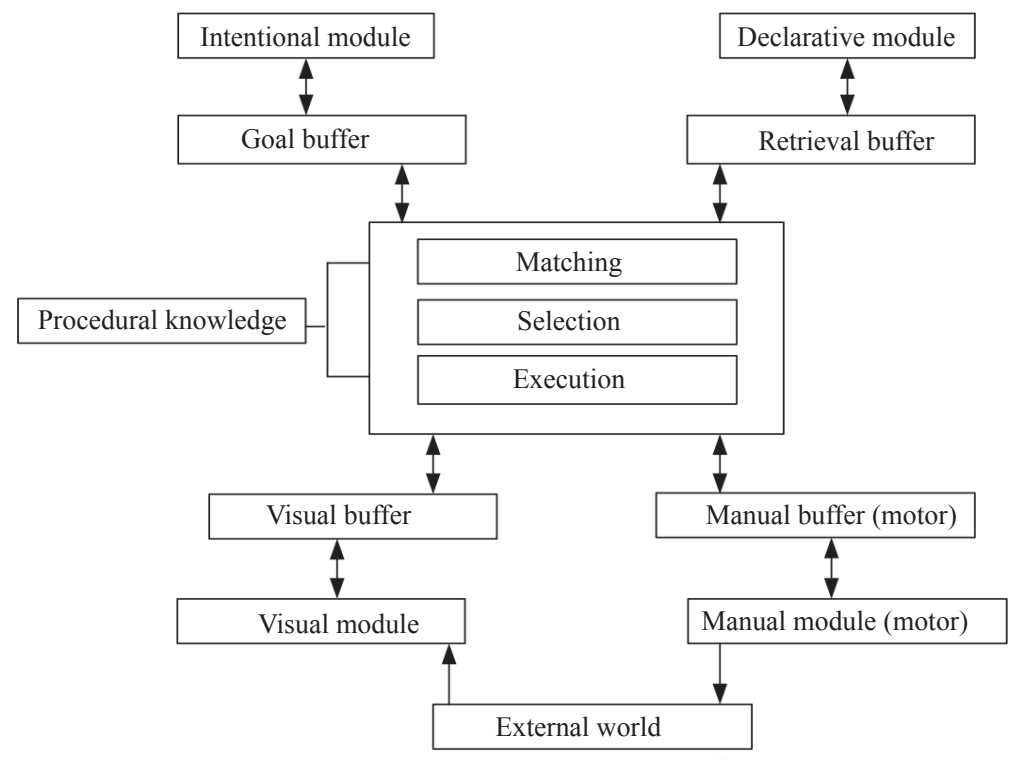

Figure 1 - Basic framework of ACT-R

to explain the things that we are aware of, and the latter is the knowledge we could display in human behaviour but that we are not conscious of [23].

Based on these assumptions, the ACT-R architecture consists of a set of modules. Each module in the ACT-R model performs a respective cognitive function and is capable of other modules independently, as shown in Figure 1.

These modules communicate with each other through the buffers. One of these modules, storing all of the chunks that represent declarative knowledge, is the declarative module. Declarative memory represents the knowledge that we already know and have learned. The procedural module is employed to hold all the procedural knowledge to decide how we behave in the world.

\section{BACKGROUND}

One view of the operators in emergencies is that the operators are capable of reading and following the steps of the emergency procedures [7]. In the metro system, the behaviour of operators under the guide of emergency procedures can also make a substantial impact on the recovering ability from a disturbed circumstance to safe operation. Alternatively, insufficient understanding and incorrect cognition of emergency procedures can weaken the emergency performance and even lead to unsafe operational recovery of the metro system.

Based on the functions automatically implemented by the system equipment, the metro system is defined into Grade of Automation (GOA) from
0 to 4 [24]. Among these GOAs, GOA 3 and GOA 4 metro systems have fully automatic train control system to drive the train, ensure safe movement of trains, and supervise the guideway, and the systems of GOA 0 to GOA 2 require a driver to accomplish the necessary train driving tasks.

With the development of the metro system automation, the emergency procedures should be adjusted correspondingly with respect to the organization structure and system configuration. Although the higher GOA system provides more automatic functions and even replaces the role of the train driver in GOA 4, the automatic functions cannot cover all the emergency scenarios, and hence, the operators in Operating Control Center (OCC) should take a more dominant position in the higher GOA systems than in the lower ones.

The emergency procedures of a metro system involve detailed guidance on how the operators should check the emergency situation, what the operators should do, and with whom the operators should communicate, such as "the traffic operator contacted the driver to confirm a fire in case of a train fire occurring between stations" in GOA 2 and "the traffic operator contacted the vehicle train attendance to confirm a fire in case of a train fire occurring between stations" in GOA 3. The behaviours of a traffic operator will be changed along with different emergency procedures, and the changes will become the key factors to affect the emergency performance. The corresponding reasonable responsibilities for different elements, such as the passengers, train, signaling system, 
Zhao B, Tang T. Evaluating the Learning Performance of Emergency Procedures for Operators in Metro Based on a Cognition...

Table 1 - Detailed responsibilities of operators in OCC

\begin{tabular}{||l|l||}
\hline Different types of operator & \multicolumn{1}{|c||}{ Responsibility } \\
\hline \hline Traffic operator & $\begin{array}{l}\text { Managing and supervising the operation such as the daily timetable, train service, train opera- } \\
\text { tion, etc. }\end{array}$ \\
\hline Environment operator & Managing and supervising electromechanical devices, water supply and drainage system. \\
\hline Power operator & Managing and supervising third rail power supply devices. \\
\hline Passenger operator & Supervising the passengers on-board and assisting passengers in emergencies. \\
\hline Vehicle operator & $\begin{array}{l}\text { Supervising the status of equipment on-board and handling the exceptional situation on-board } \\
\text { remotely. }\end{array}$ \\
\hline Maintenance operator & Receiving information of the failure and assigning the maintenance task. \\
\hline
\end{tabular}

Table 2 - Operators in different GOA system

\begin{tabular}{||l|c|c|c||}
\hline \multicolumn{1}{|c|}{ Type position } & GOA 2 & GOA 3 & GOA 4 \\
\hline \hline Traffic operator & $\sqrt{ }$ & $\sqrt{ }$ & $\sqrt{ }$ \\
\hline Environment operator & $\sqrt{ }$ & $\sqrt{ }$ & $\sqrt{ }$ \\
\hline Power operator & $\sqrt{ }$ & $\sqrt{ }$ & $\sqrt{ }$ \\
\hline Passenger operator & & & $\sqrt{ }$ \\
\hline Vehicle operator & & & $\sqrt{ }$ \\
\hline Maintenance operator & $\sqrt{ }$ & $\sqrt{ }$ & $\sqrt{ }$ \\
\hline Train driver & $\sqrt{ }$ & & \\
\hline Train attendance & & $\sqrt{ }$ & \\
\hline
\end{tabular}

station

and

infrastructure, should be assigned to the operators of different GOAs. The detailed responsibilities of operators from the role-function are summarized in Table 1. The different positions of operators indicate the different context and interactive patterns for an emergency task, and Table 2 shows the sort of operators involved in GOA 2 to 4 systems.

\section{MODEL}

Under the distinct emergency procedures of the GOA systems, it is a challenge whether the operators can understand the emergency tasks and how the operators can master the new context of the emergency-handling process. For this purpose, a cognitive model has been designed to simulate the adaptive capability of operators for learning different GOAs' emergency procedures.

Having the dominant and core role in the OCC organization, the traffic operator is modelled to focus on human performance in the emergency case of a train fire occurring between stations as a typical application. As aforementioned, the traffic operator should contact the corresponding operator or other staff to inquire about the information and make different decisions with different system configura- tions and organizational structures. Therefore, we could simplify the case of emergency procedures and select the key information that the traffic operator should know, as shown in the following items:

- When a fire alarm occurs, contact the driver to confirm whether there is a fire;

- When a fire alarm occurs, contact the driver to confirm whether the train can be moved;

- When a train fire occurs and the train can be moved, contact the driver to continuously drive the train to the next the station;

- ......

With a description of the emergency task, the traffic operator should always analyse the emergency condition and decide to contact the respective "object" to confirm the next action. For this causal relationship, the pattern of making a decision is related to three elements that are abstracted as "condition", "object" and "action". Based on this, two decision patterns are employed to simulate the decision process. As shown in Figure 2, Pattern 1 indicates that all the three elements in the imaginal module will be the retrieval indices, and Pattern 2 means that the correct decision chunk in the declarative module can be retrieved by chunks "condition" and "object" in the imaginal module.

To provide a retrieval, the chunks in the imaginal buffer spread activation to the chunks in the declarative module, and the slots in the imaginal chunks spread an amount of activation based on their relationships to other chunks in the declarative module. For example, it is assumed that the two slots which are "condition" and "object" are involved in the imaginal buffer. As a source, the two slots provide a context to retrieve the matched chunk, and therefore, the total activation $A_{i}$ of the potential chunk $i$ is defined in Equations 1 and 2 [25]: 


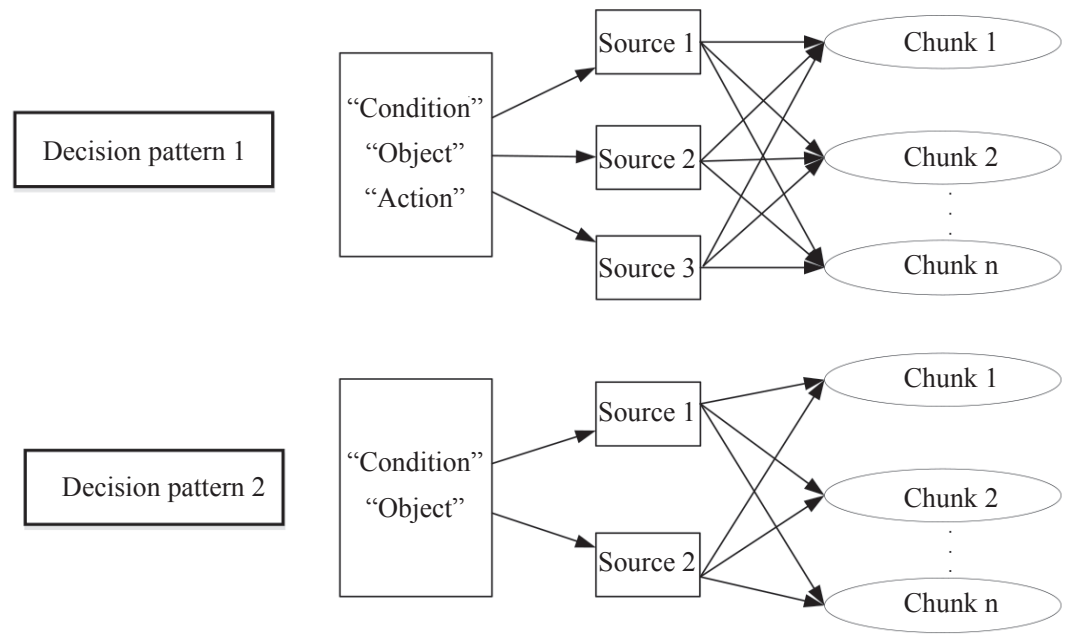

Figure 2 - Relationship between the knowledge chunk and the information source

$$
\begin{aligned}
& A_{i}=B_{i}+\sum W_{j} S_{j i} \\
& S_{j i}=S-\ln \left(\text { fan }_{j}\right)
\end{aligned}
$$

where:

$B_{i} \quad$-base-level activation of chunk $i$;

$W_{j}$-attentional weights from source $i$;

$S_{j i}$-strength of association from source $j$ to chunk $i$;

fan $_{j}$-number of chunks in declarative memory, in which $j$ is the value of a slot plus one for chunk $j$ being associated with itself;

$S$-maximum associative strength.

Total activation $A_{i}$ also determines the time of its retrieval. When a retrieval request is made, the time it takes is given by Equation 3 [25]:

Time $=F e^{-A}$

where $F$ is the latency factor; $e$ is the natural constant.

The chunk will be retrieved if its activation exceeds the retrieval threshold when a retrieval request is made. The probability of retrieval is defined in Equation 4:

$$
R P=\frac{1}{1+e^{\frac{\tau-A i}{s}}}
$$

where $\tau$ is the retrieval threshold; $s$ is the amount of noise in the system.

Parameter $S_{j i}$, which indicates the strength of association from source $j$ to chunk $i$, is calculated by Equation 2. In the activation equation, parameter $S$ is valued approximately 1.5 by Anderson and Reder [26], and we set the value as 1.6 to fit the fan data in our model. The $W_{j}$ is used to reflect the value of the imaginal activation parameter in our application; the $W_{j}$ in Pattern 1 is equal to 0.333 because there are three chunks in the imaginal buffer, and thus $W_{j}$ in Pattern 2 is equal to 0.5 . The parameter: mas is defined as 3.0 in the model.

From different GOA emergency procedures, "condition", "object" and "action" could occur in the range from one to ten times. As a result, the aim of this cognitive model was to determine the human decision patterns of the different emergency procedures of GOAs with respect to the value of fan. The logical flow chart of the cognitive model is shown in Figure 3, and the main process is as follows: attend to the content of the emergency procedures in the external interface -> Retrieve the abstract content and encode the perceptual information in the imaginal buffer $->$ Retrieve the memory module with the sources in the imaginal module -> Match the procedural rules $->$ Generate output through the motor module. Typical processes of the traffic operator range from attending to the emergency procedures to making a judgement as to which GOA it belongs.

ACT-R 7.0 was adopted to accomplish the aforementioned process. The model includes 16 production rules and 32 chunks to realize the perceptual encoding and the corresponding determining parts. The perceptual encoding part has a function that could enable the model to read the key word from the screen and store it as imaginal information, and the corresponding determining part employs the production rules on how the model makes decisions for the emergency procedures. The main production rules are listed in Table 3 [27]:

Production 1 was used to determine the location of the word such as "person" on the screen.

P1 | | ?vl | | $\mathrm{i}||->|||+\mathrm{vl}|+\mathrm{i} \mid$ | 


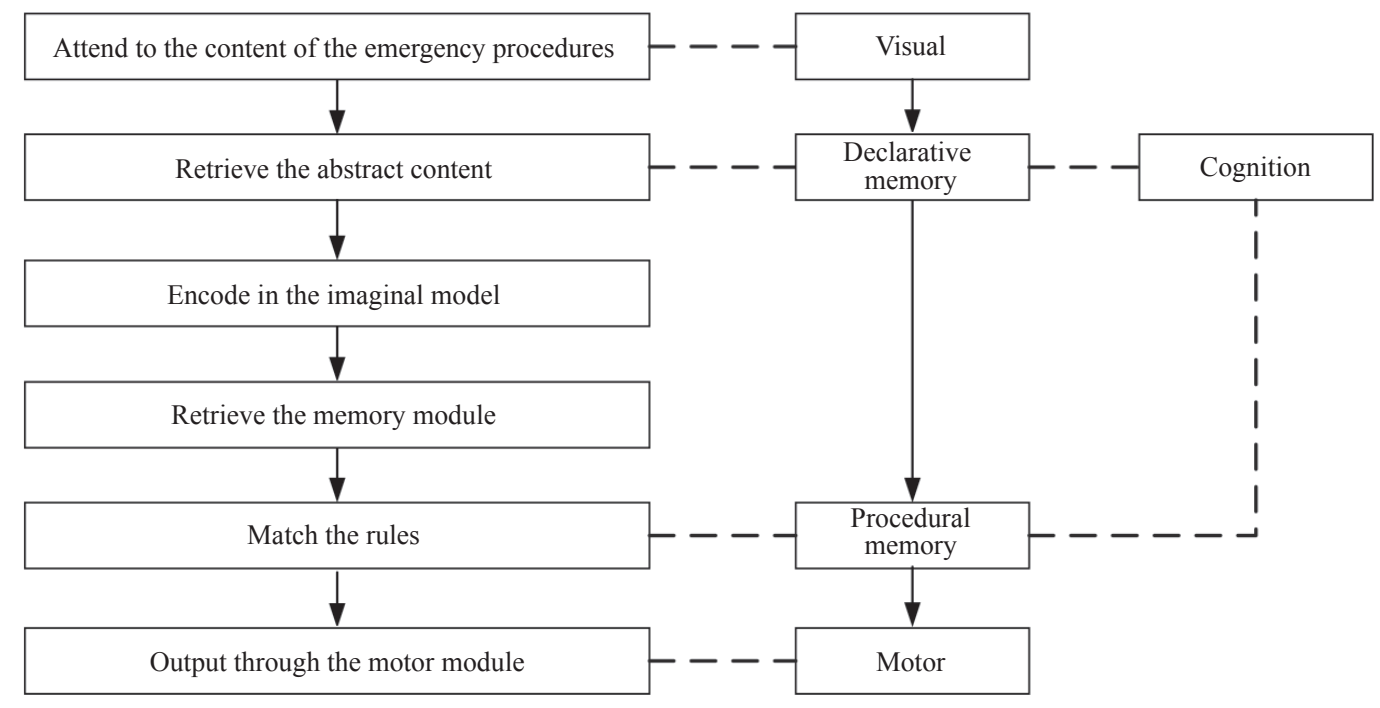

Figure 3 - Logical flowchart of the cognitive model

Table 3 - Pattern of production rules

\begin{tabular}{||c|c|c|c|c|c|c|c|c|c|c|c||}
\hline \multicolumn{9}{|c|}{ Condition } & \multicolumn{6}{c|}{ Action } \\
\hline \hline $\mathrm{g}$ & $\mathrm{r}$ & $\mathrm{v} 1(\mathrm{al})$ & $\mathrm{v}(\mathrm{a})$ & $\mathrm{i}$ & $\mathrm{m}$ & $\mathrm{g}$ & $\mathrm{r}$ & $\mathrm{v} 1$ & $\mathrm{v}$ & $\mathrm{i}$ & $\mathrm{m}(\mathrm{v})$ \\
\hline
\end{tabular}

Note: $g$ represents the goal buffer, $r$ indicates the retrieve buffer, vl means visual location buffer, i indicates the imaginal buffer, $v$ is the visual buffer, and $m$ represents the motor buffer.

Production 2 was used to request a shift of attention to the location of the word in production 1.

\section{$\mathrm{P} 2||$ |? $\mathrm{v} \mid$ ? $\mathrm{v}|||->||||+\mathrm{v}|||$}

Production 3 was used to retrieve the meaning of the word that is caught by production 2 .

\section{$\mathrm{P} 3||||=\mathrm{v}|||->||+\mathrm{r}|||||$}

Production 4 was used to place the meaning of the word into the imaginal buffer.

$$
\mathrm{P} 4||=\mathrm{r}|||=\mathrm{i}||->||||+\mathrm{v}|=\mathrm{i}||
$$

Production 5 was used to retrieve the studied emergency procedures.

P5 |?r | | =i | |->| |+r | | =i | |

Production 6 was used to retrieve a chunk and give a response that will request to the motor buffer.

$$
\text { P6 }|=\mathrm{r}|||=\mathrm{i} \mid \text { ?m }|->|||||+\mathrm{m} \mid
$$

\section{GUI OBJECT}

In most cases, the cognitive model has to interact with the external real world to obtain the information inquired by the internal logical tasks. To accomplish the interactive behaviour, the abstract representation of the external world, which is called a device, is necessary for the cognitive model.
For our proposed simulation model, an execution object based on Lisp has been designed to provide the interface for the logical model. In our case, a supervision interface was designed to simulate the detailed information about "condition", "object" and "action" in the emergency procedures. Figure 4 shows the visual window provided for the experiment of the cognitive model in which the necessary information is presented for attending to the model: when "condition"+ con "object"+ do "action". The width of the visual window is 1,000 pixels and the height of the visual window is 300 pixels.

In Figure 4 the "when" indicates the type of the emergency and its environment when it happens, the "con" denotes the dispatcher who should be involved in this emergency, and the "do" is followed by the actual tasks or the necessary actions which are implemented by the dispatcher.

In this simulation interface, the window produces the content with which the cognitive model can interact, and the cognitive model was informed to "attend" to the new refreshment, which is the change along with the information on the visual interface window. The detailed process for creating the interface is as follows: open the window $->$ clear the display $->$ present one of the emergency procedures $->$ wait for a response $->$ record the response $->$ analyse the results. 


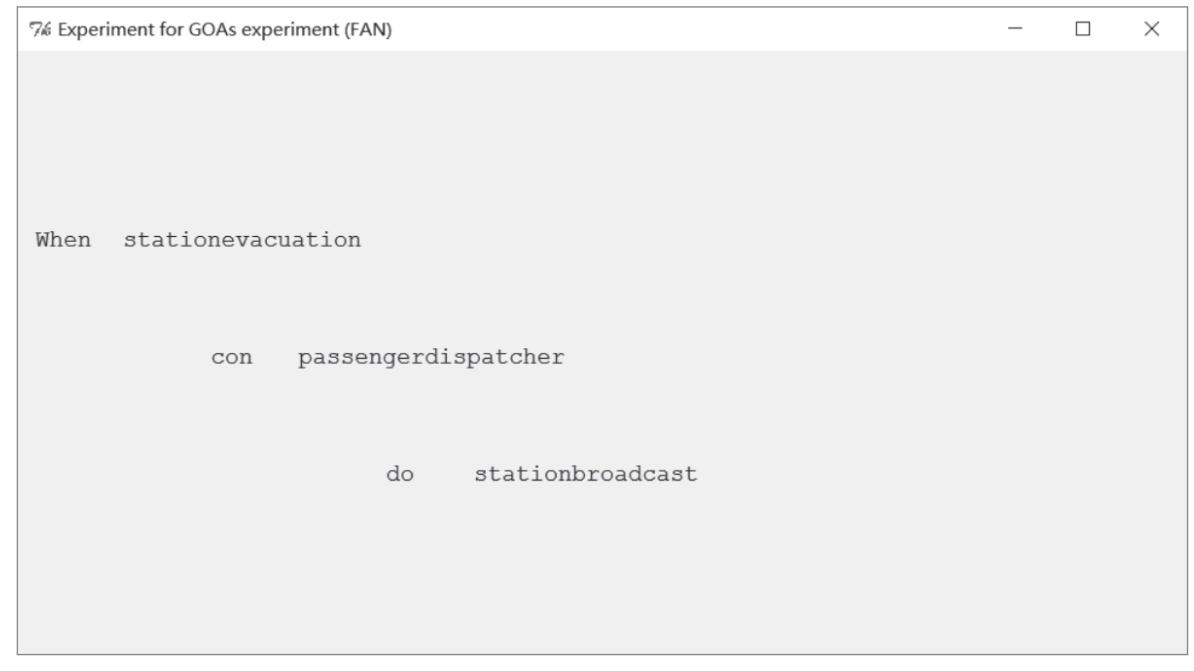

Figure 4-GUI interface for the simulation experiment

From the interface, the cognitive model can see and manipulate the corresponding information and consider it as the input parameters of the logical model. The new information is considered to be perceptual information that can be encoded in the cognitive model. With the encoded input parameters, the model can match the procedure rules during the conflicting resolution process. Then, the matching procedure with the declarative memory is preferred to determine what should happen.

\section{EXPERIMENT}

To validate the predictive results of the cognitive model in the laboratory, we designed an experiment in which 29 persons (22 students and 7 operators) participated to judge which GOA the learned emergency procedures belonged to. Among the participants, the operators were the work staff at the Beijing metro with an average working experience of five years and an age interval between 30 and 40 years, and the students were the graduate students at the National Engineering Research Center of Rail Transportation Operation and Control System and their approximate age interval was between 24 and 26 years. Both male and female participants took part (30\% female, $70 \%$ male). The emergency procedures were simplified to several knowledge tasks to facilitate the experiment process. Because some of the participants had not learned English well before, the procedures were not translated from Chinese into English and were described in Chinese. The knowledge tasks of the traffic operator in case of a train fire occurring between stations are shown in Tables 4-6.

Table 4 - Knowledge tasks of a traffic operator in case of a train fire occurring between stations (traditional GOA 2 system)

\begin{tabular}{||ll||}
\hline 1. & If a fire alarm occurs, contact the driver to confirm whether there is a fire. \\
\hline 2. & If a fire alarm occurs, contact the driver to confirm whether the train can be moved. \\
\hline 3. & $\begin{array}{l}\text { If an uncontrolled fire and unmovable train are confirmed, contact the driver to guide passengers into an evacuation } \\
\text { section. }\end{array}$ \\
\hline 4. & If a controlled fire and unmovable train are confirmed, contact the driver to control the fire. \\
\hline 5. & If a controlled fire and unmovable train are confirmed, contact the driver to wait for rescue. \\
\hline 6. & If a fire and movable train are confirmed, contact the driver to move the train to the next station. \\
\hline 7. & If a fire is not confirmed, contact the driver to move the train to the station. \\
\hline 8. & If a fire is not confirmed, (traffic operator) cancel the fire alarm. \\
\hline 9. & If station evacuation occurs, contact the driver to broadcast on-board. \\
\hline 10. & If station evacuation occurs, contact the driver to open the train door in the station. \\
\hline 11. & If station evacuation occurs, contact station staff to guide passengers into station \\
\hline 12. & If station evacuation occurs, (traffic operator) hold the train at the next station. \\
\hline
\end{tabular}


Table 5 - Knowledge tasks of a traffic operator in case of a train fire occurring between stations (GOA 3 system)

\begin{tabular}{|ll||}
\hline 1. & If a fire alarm occurs, contact the train attendant to confirm whether there is a fire. \\
\hline 2. & If a fire alarm occurs, contact the train attendant to confirm whether the train can be moved. \\
\hline 3. & $\begin{array}{l}\text { If an uncontrolled fire and unmovable train are confirmed, contact the train attendant to guide passenger into an } \\
\text { evacuation section. }\end{array}$ \\
\hline 4. & If an uncontrolled fire and unmovable train are confirmed, contact the train attendant to control the fire. \\
\hline 5. & If a controlled fire and unmovable train are confirmed, contact the train attendant to wait for rescue. \\
\hline 6. & If a fire and movable train are confirmed, (traffic operator) confirm the train should move to the next station. \\
\hline 7. & If a fire is not confirmed, (traffic operator) cancel the fire alarm. \\
\hline 8. & If station evacuation occurs, contact the train attendant to broadcast on-board. \\
\hline 9. & If station evacuation occurs, contact the station staff to guide passengers into station evacuation. \\
\hline 10. & If station evacuation occurs, (traffic operator) hold the train at the next station. \\
\hline \hline
\end{tabular}

Table 6 - Knowledge tasks of a traffic operator in case of a train fire occurring between stations (GOA 4 system)

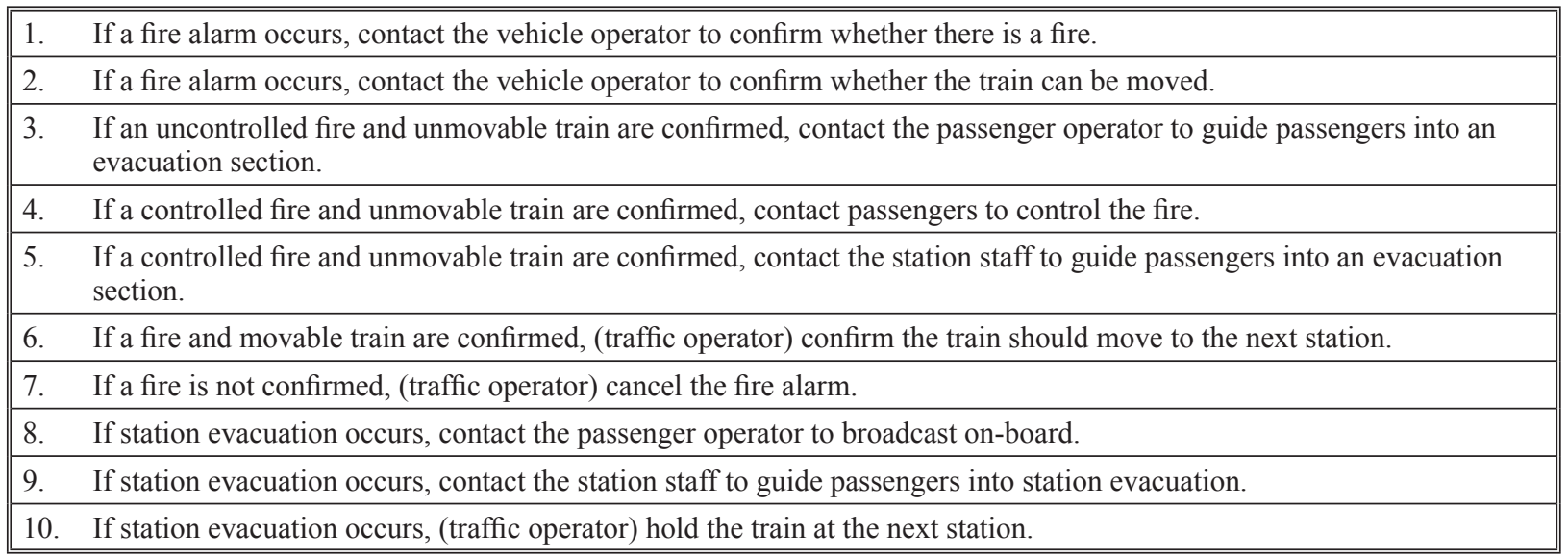

Table 4 is learned by the participants to memorize the knowledge of the traditional system (GOA 2). After that, the knowledge tasks of higher levels of automated metro systems (GOA 3 and GOA 4) are further learned to form the respective memory, as shown in Tables 5 and 6 .

After that, the participants are required to judge which GOA system the knowledge task belongs to. Six sets of knowledge tasks are chosen to test the decision process of the participants. Each knowledge task in the same set has the same condition but different objects and actions in different GOAs. The time interval of participants from checking the knowledge tasks to outputting the levels of GOA is recorded.

In conclusion, the process of the cognitive experiment about knowledge tasks is shown as follows:

1) Learn Tables 4-6 within 1 minute for each table and the initial interval is 60 seconds.

2) Judge the test item (knowledge task in Tables 4-6) to which GOA it belongs.

3) Inform the participants whether the answers are all correct.
4) Repeat steps 1 to 3 until the results of the test are all correct. The time for each relearning will be reduced by 10 seconds.

\section{SIMULATION RESULTS AND DISCUSSIONS}

To reveal the process of how the operators understand these emergency procedures of different GOAs, the simulation data were compared with the experimental data.

As shown in Figure 5, x-axis denotes the tested emergency procedure rule number, and y-axis represents the responsive time of the participant. The blue dots with broken lines denote the simulation data, and the red dots represent the actual data from the experiment.

Table 7 shows the correlation coefficient of each procedure rule between the cognitive model and the experiment. From the table, most correlation coefficient values of procedure rule numbers are over 0.9 , and only one procedure rule correlation coefficient value is between 0.8 and 0.9 , which indicates that the results of the proposed cognitive model have 
Zhao B, Tang T. Evaluating the Learning Performance of Emergency Procedures for Operators in Metro Based on a Cognition...

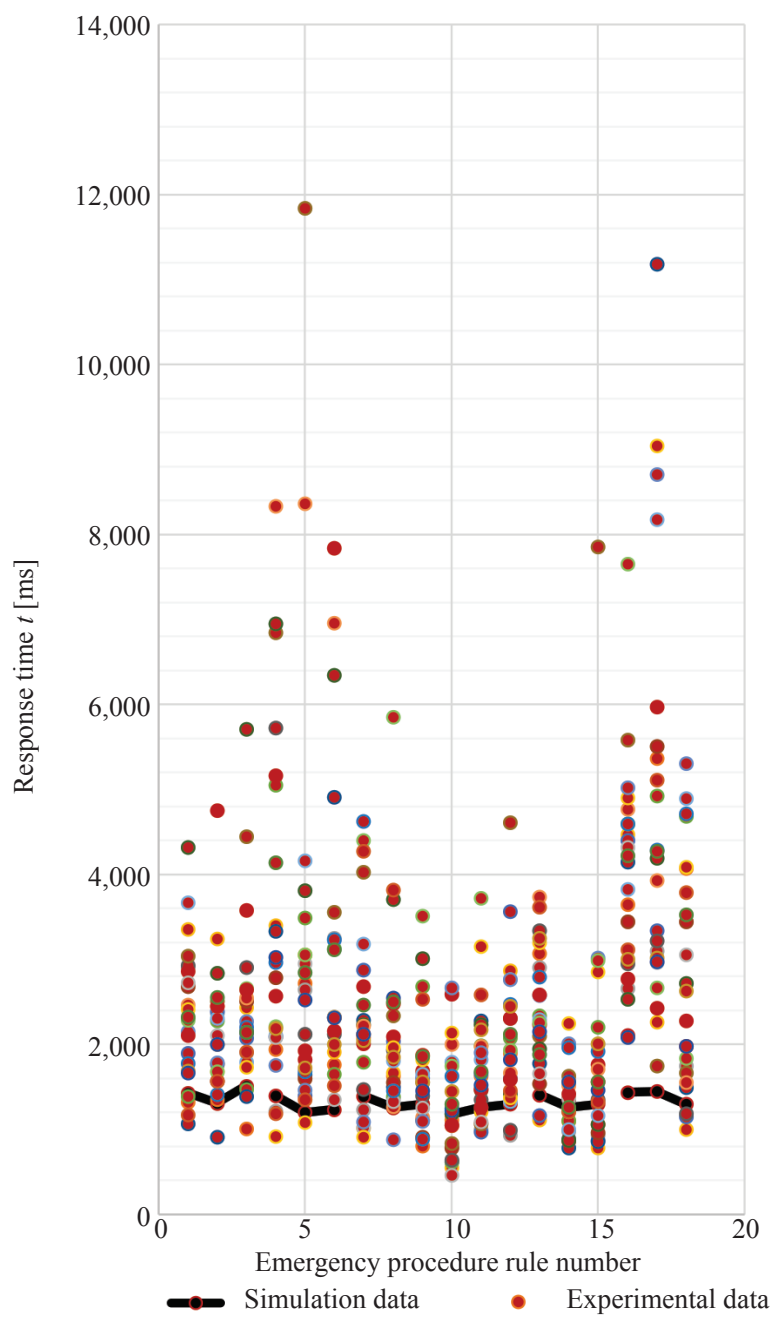

Figure 5 - Comparison of model data and experimental data
Table 7 - Correlation coefficients for procedure rules

\begin{tabular}{|c|c|}
\hline Procedure rule number & Correlation coefficient \\
\hline \hline P1 & 0.989936 \\
\hline P2 & 0.97793 \\
\hline P3 & 0.815066 \\
\hline P4 & 0.994591 \\
\hline P5 & 0.98859 \\
\hline P6 & 0.904772 \\
\hline
\end{tabular}

high correlation with the results of the experiment. The simulation results are expected to have high correlation with the experimental study at an optimal fitting level.

Based on the simulation process and the experiment of the participants, the decision pattern of each procedure rule with the fan value is identified to demonstrate the human cognitive performance of an emergency decision. Tables 8-10 show the recognition latencies for six procedure rules of different GOAs from the simulation, and each procedure rule has its respective decision pattern for "condition", "object" and "action" fan values. For most knowledge tasks, the recognition delays of GOA 3 could be shorter than those of GOA 2 and GOA 4.

From Tables 8-10, the effect of fan is the factor that affects the recognition latencies, and at the same time, the decision pattern has a positive influence on the response time. Decision pattern 1 is the main

Table 8 - Cognition delays for the traditional GOA 2 system

\begin{tabular}{|c|c|c|c|c|c||}
\hline $\begin{array}{c}\text { Procedure rule } \\
\text { number }\end{array}$ & $\begin{array}{c}\text { Decision } \\
\text { pattern }\end{array}$ & “condition” Fan & “object Fan & “action”Fan & Time [ms] \\
\hline \hline P1 & 1 & 10 & 9 & 2 & 1,509 \\
\hline P2 & 2 & 3 & 9 & 3 & 1,233 \\
\hline P3 & 2 & 6 & 9 & 3 & 1,297 \\
\hline P4 & 2 & 6 & 9 & 3 & 1,297 \\
\hline P5 & 2 & 6 & 9 & 3 & 1,297 \\
\hline P6 & 2 & 6 & 9 & 3 & 1,297 \\
\hline
\end{tabular}

Table 9 - Cognitive delays for the GOA 3 system

\begin{tabular}{||c|c|c|c|c|c||}
\hline $\begin{array}{c}\text { Procedure } \\
\text { rule number }\end{array}$ & $\begin{array}{c}\text { Decision } \\
\text { pattern }\end{array}$ & “condition” Fan & “object” Fan & "action” Fan & Time [ms] \\
\hline \hline P1 & 2 & 10 & 6 & 3 & 1,310 \\
\hline P2 & 2 & 3 & 6 & 3 & 1,201 \\
\hline P3 & 1 & 6 & 6 & 3 & 1,255 \\
\hline P4 & 2 & 6 & 6 & 3 & 1,255 \\
\hline P5 & 2 & 6 & 6 & 3 & 1,255 \\
\hline P6 & 1 & 6 & 6 & 3 & 1,436 \\
\hline
\end{tabular}


Zhao B, Tang T. Evaluating the Learning Performance of Emergency Procedures for Operators in Metro Based on a Cognition...

Table 10 - Cognitive delays for the GOA 4 system

\begin{tabular}{||c|c|c|c|c|c||}
\hline $\begin{array}{c}\text { Procedure } \\
\text { rule number }\end{array}$ & $\begin{array}{c}\text { Decision } \\
\text { pattern }\end{array}$ & “condition” Fan & “object” Fan & "action” Fan & Time [ms] \\
\hline \hline P1 & 1 & 10 & 2 & 2 & 1,430 \\
\hline P2 & 1 & 3 & 2 & 3 & 1,393 \\
\hline P3 & 1 & 6 & 1 & 3 & 1,390 \\
\hline P4 & 2 & 6 & 2 & 3 & 1,179 \\
\hline P5 & 1 & 6 & 2 & 3 & 1,407 \\
\hline P6 & 1 & 6 & 4 & 3 & 1,445 \\
\hline \hline
\end{tabular}

cognitive approach for GOA 4 system, but for GOA 2 and GOA 3 systems, Pattern 2 rather than Pattern 1 plays the dominant role.

Among these participants, most of the operators prefer Pattern 1 for GOA 4 system and GOA 3 system; also, they use both Pattern 2 and Pattern 1 for GOA 2 system. Furthermore, their experimental data are shorter than the average data from student participants.

However, the variance of the response time between the simulation model and the experiment indicates that the parameters in ACT-R model need to be optimized. Particularly, some distinct different data are caused by individual and emotional factors, and it is difficult to simulate the disturbing cases in the ACT-R environment. In addition, the fixed areas are employed to attend to the knowledge of the ACT-R model, but the areas for procedural rules in the actual testing environment are variable.

\section{CONCLUSION}

The proposed model accounts for the visual awareness, hand output and memory-related decision-making to simulate the different levels of an operator's learning behaviour. The perceptual-motor process to simulate how the operators focus on emergency procedures is close to practical learning scenarios. Also, the data comparison of the simulation and the experimental study indicated that the quantitative analysis was nicely available in the cognitive model. A prediction for the cognitive capability of the learning emergency procedures can provide feedback for the optimal design of operational guidance and procedures. The recognition delays are related to the fan value of the basic knowledge in emergency procedures and the pattern by which the decision is made. The recognition delays of emergency procedures in GOA 4 are more than that of other lower GOAs under the same condi- tions. The prediction results for the operator's cognitive capability indicate fewer new positions will lead to less recognition delays.

Modelling operator behaviours in cognitive architectures could be considered as a beneficial approach for automated metro about how the evolution of GOAs affects the staff training. Potential applications include the exploration of the higher-level cognitive activities of operators and the evaluation of the human-machine interface in metro.

Further study will focus on a deliberate cognitive approach to explore the higher-level cognitive activities of operators in an automated metro system, and more research will be done on how feedback of the cognitive model influences the emergency procedures in emergency scenarios.

\section{ACKNOWLEDGEMENTS}

This work was supported by the Beijing Laboratory of Urban Rail Transit, Beijing Key Laboratory of Urban Rail Transit Automation and Control, and the National Natural Science Foundation of China (No. 61790573).

赵波波, 通讯作者, 北京交通大学 轨道交通运行控制 系统国家工程研究中心。北京市海淀区西直门外上园 村3号。邮编: 100044. 邮箱: bbzhao@bjtu.edu.cn 唐涛, 北京交通大学 轨道交通控制与安全国家重 点实验室。北京市海淀区西直门外上园村3号。邮 编: 100044. 邮箱: ttang@bjtu.edu.cn

\section{基于认知模型评价地铁调度员的应急规程学习 性能}

摘要

随着地铁自动化程度的快速发展, 有效的应急 规程在地铁应急响应和修复过程中起到了一个至关 重要的作用, 同时地铁调度员面临了学习各种自动 化等级下地铁应急规程的挑战。因此, 本论文旨在 评价调度员在各种应急规程下的学习性能。基于 $A C T-R$ 认知理论, 针对调度员应急规程学习过程提 出了两种决策模式, 设计的认知模型包含了 16 种产 
生式规则和 32 个知识块, 进一步构建感知编码和相 应的决策确定模块, 最后通过实验验证了模型结 果。

\section{关键词}

认知模型; 应急规程; 人因性能建模; 轨 道交通系统; 自动化等级; 自动化信任

\section{REFERENCES}

[1] Bainbridge L. Ironies of automation. Analysis, design and evaluation of man-machine systems. 1982;15(6): 129135.Available from: doi:10.1016/B978-0-08-0293486.50026-9

[2] Steinkohl J, Perreal Y. SECUR-ED: Providing a set of tools to improve urban transport security. Eurotransport. 2012;10: 74-76. Available from: https://www.intelligenttransport.com/wp-content/uploads/Security-Supplement-2012.pdf [Accessed $5^{\text {th }}$ September 2018].

[3] Bruyelle JL, O’Neill C, El-Koursi EM, Hamelin F, Sartori N, Khoudour L. Improving the resilience of metro vehicle and passengers for an effective emergency response to terrorist attacks. Safety science. 2014;62: 37-45. Available from: doi:10.1016/j.ssci.2013.07.022

[4] Feyer AM, Williamson AM, Cairns DR. The involvement of human behavior in occupational accidents: Errors in context. Safety Science. 1997;25(1): 55-65. Available from: doi:10.1016/S0925-7535(97)00008-8

[5] Rouse SH, Rouse WB, Hammer JM. Design and evaluation of an onboard computer-based information system for aircraft. IEEE Transactions on Systems, Man, and Cybernetics. 1982;12(4): 451-463. Available from: doi:10.1109/TSMC.1982.4308849

[6] Hollnagel E. Human reliability assessment in context. Nuclear Engineering and Technology. 2015; 37(2): 159166. Available from: http://www.koreascience.or.kr/ article/JAKO200502637315489.page [Accessed $5^{\text {th }}$ September 2018].

[7] Roth EM, Mumaw RJ, Lewis PM. An empirical investigation of operator performance in cognitively demanding simulated emergencies. Nuclear Regulatory Commission, Div. of Systems Research, Westinghouse Electric Corp, Science and Technology Center. Report number: NUREG/CR-6208, 1994.

[8] Embrey D, Kontogiannis T, Green M. Guidelines for preventing human error in process safety. New York: Center for Chemical Process Safety; 1994.

[9] Kernan MC, Bruning NS, Miller-Guhde L. Individual and group performance: Effects of task complexity and information. Human Performance. 1994;7(4): 273-289. Available from: doi:10.1016/j.chb.2008.12.008

[10] Brune RL, Weinstein M. Development of a checklist for evaluating maintenance, test and calibration procedures used in Nuclear Power Plants. Sandia National Labs, Human Performance Technologies, Inc. Report number: NUREG/CR-1368; SAND-80-7053, 1980.

[11] Brune RL, Weinstein M. Development of a checklist for evaluating emergency procedures used in nuclear power plants. Human Performance Technologies, Inc. Report number: NUREG/CR-1970; SAND-81-7070, 1981.

[12] Brune RL, Weinstein M. Checklist for evaluating emergency operating procedures used in nuclear power plants. Human Performance Technologies, Inc. Report number: NUREG/CR - 2005; SAND-81-7074, 1983.

[13] Garner JD, Blethrow JG. Evacuation tests from an SST mock-up. Civil Aerospace Medical Institute. Report number: AM 70-19, 1970.

[14] Park J, Jung W. A study on the validity of a task complexity measure for emergency operating procedures of nuclear power plants-Comparing task complexity scores with two sets of operator response time data obtained under a simulated SGTR. Reliability Engineering \& System Safety. 2008;93(4): 557-566. Available from: doi: 10.1016/j.ress.2007.02.002

[15] Park J, Jung W, Jung K. The effect of two complexity factors on the performance of emergency tasks - An experimental verification. Reliability Engineering \& System Safety. 2008;93(2): 350-362. Available from: doi: 10.1016/j.ress.2006.09.027

[16] Dien Y. Safety and application of procedures, or 'how do 'they' have to use operating procedures in nuclear power plants?'. Safety Science. 1998;29(3): 179-187. Available from: doi:10.1016/S0925-7535(98)00021-6

[17] Salvucci DD. Modeling driver behavior in a cognitive architecture. Human Factors: The Journal of the Human Factors and Ergonomics Society. 2006;48(2): 362-380. Available from: doi:10.1518/001872006777724417

[18] Nuclear Regulatory Commission. Guidelines for the preparation of emergency operating procedures. Nuclear Regulatory Commission. Report number: NUREG-0899, 1982.

[19] Nuclear Regulatory Commission. Loss of main and auxiliary feedwater event at the Davis-Besse plant on June 9, 1985. Nuclear Regulatory Commission. Report number: NUREG-1154, 1985.

[20] Nuclear Regulatory Commission. Transformer failure and common-mode loss of instrument power at Nine Mile Point Unit 2 on August 13, 1991. Nuclear Regulatory Commission. Report number: NUREG-1455, 1991.

[21] Byrne MD, Kirlik A. Using computational cognitive modeling to diagnose possible sources of aviation error. International Journal of Aviation Psychology. 2005;15: 135155. Available from: doi:10.1207/s15327108ijap1502_2

[22] Anderson JR. The architecture of cognition. Cambridge, MA: Harvard University Press; 1983.

[23] Anderson JR, Matessa M, Lebiere C. ACT-R: A theory of higher level cognition and its relation to visual attention. Human-Computer Interaction. 1997;12(4): 439-462. Available from: doi:10.1207/s15327051hci1204_5

[24] International Electrotechnical Commission. IEC 622902:2011. Railway applications - Urban guided transport management and command/control systems, Part 2: Functional requirements specification. Geneva: IEC; 2011.

[25] Anderson JR, Bothell D, Byrne MD, Douglass S, Lebiere C, Qin Y. An integrated theory of the mind. Psychological Review. 2004;111(4): 1036-1060. Available from: doi:10.1037/0033-295x.111.4.1036

[26] Anderson JR, Reder LM. The fan effect: New results and new theories. Journal of Experimental Psychology General. 1999;128: 186-197. Available from: doi:10.1037//0096-3445.128.2.186

[27] Kim JW. Procedural skills: From learning to forgetting. $\mathrm{PhD}$ thesis. The Pennsylvania State University; 2008. 